\title{
The Primary Olfactory Projection Has Two Chemically Distinct Zones
}

\author{
James E. Schwob and David I. Gottlieb \\ Department of Anatomy and Neurobiology, Washington University School of Medicine, St. Louis, Missouri 63110
}

The sensory neurons of the olfactory epithelium form an anatomically uniform population but are differentially excited by odorants. We have discovered an unexpected biochemical heterogeneity within this population that extends to its axonal projection onto the olfactory bulb. This heterogeneity is recognized by a newly generated monoclonal antibody, designated RB-8, that differentially stains the primary olfactory projection in rats and divides it into 2 nonoverlapping zones.

With light-microscopic immunohistochemistry, RB-8 densely labels the fascicles of the olfactory nerve from the ventral and lateral parts of the olfactory epithelium, where there is also some epithelial staining. This area, which we designate RB-8positive, comprises about two-thirds of the epithelial sheet. RB-8 labeling of the other third of the epithelium, which includes the dorsal recess and medial tips of the dorsal turbinals, is not detectable, and the fascicles from these RB-8-negative areas are only weakly stained. These RB-8-negative areas form a contiguous zone on flattened maps of the epithelial sheet. In the olfactory bulb, RB-8 staining of the glomeruli in the ventrolateral part is correspondingly dense, while that in the dorsomedial glomeruli is undetectable or very light. In the labeled glomeruli, the RB-8 staining is precisely coextensive with anti-olfactory marker protein staining, which serves as a marker for the olfactory axons and terminals. In addition, knife-cut lesions of the olfactory nerve totally eliminate the RB-8 staining in the glomeruli where the destruction of the olfactory terminals is complete. There is also a good correlation between the staining patterns in the bulb and epithelium and what is known from tract-tracing studies of the arrangement of the axonal projection of the epithelium onto the bulb. This evidence strongly suggests that, in the olfactory nerve and glomeruli, RB-8 stains the olfactory axons and their terminals. A survey of the CNS and peripheral tissues demonstrates that staining with RB-8 is nervous system-specific; not all components of the CNS and PNS are stained.

The antigen recognized by $\mathrm{RB}-8$ was characterized in immunoblots and by use of a direct radioimmunoassay (RIA) which assessed binding of ${ }^{125}$ I-RB-8. With this assay, the RB-8 binding sites in whole brain are shown to be membrane-associated, saturable, immunologically specific for RB-8, and trypsin-sensilive. In SDS-PAGE immunoblots of membrane proteins, the antigen in rat forebrain and in the olfactory nerve is a protein of $\mathbf{1 2 5}$ kDa $M_{r}$, which comigrates in mixtures of membranes from the 2 sources. Thus, the antigen in the olfactory nerve is likely to

Received Jan. 27, 1986; revised Apr. 22, 1986; accepted Apr. 24, 1986

The authors would like to thank Jacqueline E. Snyder, Nuri B. Farber, and Robert Mead for technical assistance in the completion of this work. We would also like to thank Dr. Frank Margolis for his generous gift of the antiserum to olfactory marker protein. We acknowledge the thoughtful comments and criticisms of Drs. Karina Meiri, Joseph L. Price, Gordon Shepherd and Mark Willard during the course of this project and on this manuscript. This work was supported by NIH Grant NS 12867; J.E.S. was supported by NIH Training Grants NS 07076 and NS 07057.

Correspondence should be addressed to Dr. James E. Schwob at the above address.

Copyright (C) 1986 Society for Neuroscience $0270-6474 / 86 / 113393-12 \$ 02.00 / 0$ be identical or similar to the antigen found elsewhere in the CNS.

These results demonstrate that the primary olfactory projection has 2 nonoverlapping zones; one of these is stained well with RB-8 and the other is not. Since the responsivity to odorants in the olfactory epithelium and bulb is nonhomogeneously distributed, the RB-8-positive and negative zones may have distinguishable functional roles in olfaction.

In vertebrates, the olfactory sensory neurons, which reside in the olfactory epithelium lining the posterodorsal part of the nasal cavity, are differentially activated by odorants. The types of odorant receptor molecules expressed by this sensory population have not been enumerated or classified, and progress in their biochemical identification is comparatively recent (reviewed by Lancet, 1986). However, there is substantial evidence for a degree of spatial organization in olfactory sensory coding by the epithelium, which is maintained in the axonal projection onto the glomeruli of the olfactory bulb. The evidence for a spatial component includes the finding of regional differences in the responsivity of the olfactory epithelium to a variety of odorants (Lancet et al., 1981; Mackay-Sim et al., 1982; Mozell, 1964), and of punctate, regionally restricted activation of the olfactory bulb by selected compounds (Adrian, 1953, 1954; Jourdan et al., 1980; Lancet et al., 1982; Skeen, 1977; Stewart et al., 1979).

We have recently generated a mouse monoclonal antibody, designated RB-8, that recognizes a biochemical difference hetween parts of the olfactory epithelium and its axonal projection and divides them into 2 broad, nonoverlapping zones. One zone, termed RB-8-positive, is located ventral and lateral in the epithelium and the bulb. In this zone, the fascicles of the olfactory nerve and the glomeruli of the bulb stain heavily with the antibody. The complementary zone, termed RB-8-negative, is located dorsal and medial. These structures are stained poorly, if at all, with RB-8. The antigen recognized by $\mathrm{RB}-8$ is a membrane-associated protein of $125 \mathrm{kDa} M_{\mathrm{r}}$. The ability of this monoclonal antibody to distinguish 2 spatial zones in the olfactory epithelium and the evidence for a spatial component to olfactory coding suggest that the RB- 8 antigen and this division of the primary olfactory projection play an important, although as yet undefined, functional role. A preliminary report of this work has already been published (Schwob and Gottlieb, 1985).

\section{Materials and Methods}

\section{Animals}

Six- to 8-week-old Sprague-Dawley rats were obtained from a barrierisolated facility (Sasco, Inc.). All animals used were free of chronic rhinitis or epithelial atrophy, as assessed by light-microscopic examination. The neonatal rats used as a source of immunogen were sacrificed within $24 \mathrm{hr}$ of birth. Balb/c mice were obtained from Cumberland View Farms (Clinton, TN).

\section{Materials}

All biochemical and immunohistochemical reagents were reagent grade and obtained from Sigma Chemical Co. unless otherwise specified. 


\section{Monoclonal antibody production}

$\mathrm{RB}-8$, a mouse monoclonal $\mathrm{IgG}_{1}$ antibody, was generated by the fusion of SP 2/0-Ag 14 mouse mycloma cells (American Type Culture Collection) with splenocytes from a Balb/c mouse that had been previously immunized with intraperitoneal injections of a suspension of neonatal rat piriform cortex. The mouse was immunized 4 times over a 10 week period. Seventy-two hours after the last immunization, the splenocytes were fused using standard methods (Galfre et al., 1977). Selection of the RB-8-secreting hybridoma was based on its pattern of staining of cryostat sections of weakly fixed brain from adult and neonatal rats. It was cloned twice by limiting dilution and was found to be a stable producer of immunoglobulin on the basis of repeated morphological screenings of supernatants from the clonal line over several months of continuous growth. Ascites containing RB-8 was produced in $\mathrm{Balb} / \mathrm{c}$ mice.

\section{Indirect light-microcopic immunohistochemistry}

Tissue for immunohistochemistry was prepared as described in Schwob et al. (1986). A variety of fixation methods was evaluated for the preservation and consistency of the staining pattern. These included (1) using fresh-frozen tissue from the olfactory bulb, olfactory epithelium, and forebrain; (2) perfusion fixation with $0.5-4 \%$ buffered paraformaldehyde, with paraformaldehyde-glutaraldehyde mixtures $(0.1-0.5 \%$ glutaraldehyde), or with paraformaldehyde in combination with 0.075 M lysine and 0.01 M sodium periodate (McLean and Nakane, 1974); and (3) postsectioning fixation of fresh-frozen tissue with acetone, absolute alcohol, or a 1:1 chloroform-methanol mixture. In each case, qualitatively similar results were obtained, although the staining intensity was decreased by higher concentrations of paraformaldehyde or glutaraldehyde. The best overall preservation of antigenicity and tissue structure was achieved by perfusion fixation with cold $0.01 \mathrm{M}$ sodium periodate, $0.075 \mathrm{M}$ lysine, and $0.5-1.0 \%$ paraformaldehyde in $\mathrm{pH} 7.2$ phosphate buffer. Subsequent preparation of the tissue was according to Schwob et al. (1986).

Frozen semithin sections of the olfactory bulb were cut at a thickness of $0.5-0.7 \mu \mathrm{m}$ on a Sorvall MT-2 ultramicrotome equipped with a Sorvall low-temperature sectioning device. Blocks of the ventral half of the olfactory bulb, 1-2 mm thick, were equilibrated for $6 \mathrm{hr}$ in $30 \%$ sucrose before rapid freezing in liquid nitrogen, and were stored at $-70^{\circ} \mathrm{C}$ before sectioning. Sections were collected onto coverslips, spread under a drop of saturated sucrose, and stored at $-20^{\circ} \mathrm{C}$ before staining.

Immunohistochemical staining by the avidin-biotinylated HRP method (Vectastain; Vector Laboratories) was performed according to the procedure of Schwob et al. (1986). Primary reagents were used at the following concentrations: RB-8 ascites, 1:1000; normal mouse serum, 1:1000 (both were diluted in PBS with $20 \%$ normal horse serum and $0.2 \%$ Triton $X-100$ ); goat antiserum to rat olfactory marker protein (the gift of Dr. Frank Margolis), 1:1200; or normal goat serum, 1:1200 (both were diluted in PBS with 20\% normal rabbit serum). Frozen thin sections of the olfactory bulb were double-labeled with $\mathrm{KB}-8$ and antiolfactory marker protein with the following modification of the standard protocol: The tissue was sequentially incubated for $30 \mathrm{~min}$ in $\mathrm{RB}-8$ ascites at 1:200, fluoresceinated rabbit anti-mouse IgG (Dako) at 1:20, anti-olfactory marker protein at 1:200, biotinylated rabbit anti-goat IgG (Vector) at 1:150, and rhodaminated avidin (Vector) at 1:300. Each was diluted in PBS-20\% normal rabbit serum. The sections were coverslipped with $p$-phenylenediamine-containing glycerol (Johnson and Nogueria Araujo, 1981) and photographed with a Leitz Dialux 22 equipped with a $50 \mathrm{~W}$ mercury lamp and Leitz L2 (fluorescein-specific) and N2 (rhodamine-specific) filters. Control experiments omitting either the mouse- or goal-specific secondary reagents demonstrated that there was no species cross-reactivity of the secondary reagents, and that the fluorescent signal was transmitted only by the corresponding specific filter.

\section{Two-dimensional reconstructions of the olfactory epithelium and olfactory bulb}

A flattened map of the epithelial sheet (Fig. 2) was constructed from low-power photomicrographs of a series of coronal sections of the olfactory epithelium by adapting the procedure used by Van Essen and Maunsell (1980) to make flattened reconstructions of the primate cerebral cortex. In both cases, the aim was to represent accurately a highly folded 2-dimensional surface that bends through 3 dimensions so that points that are near one another in the animal would remain neighbors in the flattened representation. With the olfactory epithelium, this was particularly difficult near its caudal limit. Posteriorly, the lateral wall of the nasal cavity and the septum fuse to form the back wall, and there are epithelial-lined cul-de-sacs that project further posteriorly to surround the lateral and ventral surfaces of the olfactory bulb. Here, the configuration of the epithelial surface around the bulb is analogous to that of a balloon surrounding objects that indent it. In order to accommodate this spatial relationship and to minimize distortion, the following procedure was employed: The caudalmost coronal section in which the olfactory epithelium can be traced as a continuous line (level 2 in Fig. 2) was plotted first. Linear distortion was eliminated for this and all other sections by plotting the epithelial surface as a curved line that mapped to scale the exact distance along the surface between defined landmarks, such as the tips of the turbinals (e.g., points "a-g" of level 2 in Fig. 2). The curvature of this line was adjusted so that the points that fuse together in the animal to form the back wall of the nasal cavity were drawn together near the center of the map. For example, points "d-f" of level 2 in Figure 2 are on the tips of separate but adjacent turbinals in the coronal plane. However, they fuse together between levels 1 and 2 and are contiguous in a perisagittal plane. The mapping procedure restored the neighbor relationship between these points. The lateral parts of the epithelium, which are tightly curled in the animal, were unfolded into simple loops. At the dorsal recess, the contour line had to be bent back nearly $180^{\circ}$ to accommodate this unfolding. These distortions in angular relationships were necessary to allow the contour lines representing adjacent sections to be added to the map with equal spacing. The epithelial cul-de-sacs situated posterior to this level (e.g., level 1 in Fig. 2) were nested inside of these loops toward the center of the drawing. More anterior levels (e.g., level 3 in Fig. 2) were drawn as successive layers around the reference level, taking care to align the tips of the turbinals (points "a-f") and the dorsal recess (point "g") for each level. This procedure minimized distance errors between points in a single section or in adjacent sections, allowing a more accurate comparison of surface areas between different parts of the epithelium.

In contrast, the glomerular surface of the olfactory bulb is essentially a smooth cylinder, which can be split along the ventrolateral comer of the bulb, and unrolled to form a flattened, 2-dimensional map. Thus, each of a series of coronal sections through the bulb was mapped as a straight line that was separated from the other straight-line representations by the distance between the sections (Fig. 6).

\section{Lesions of the olfactory nerve}

The fascicles of the olfactory nerve on one side were severed extracranially by anesthetizing 2 adult rats with $7 \%$ chloral hydrate and removing the frontal bone rostral to the tip of the olfactory bulb to expose the olfactory mucosa. A flat probe was inserted on the nasal side of the cribriform plate near the midline and pushed ventrally and laterally to sever the nerve. After surgery, the animals survived for 2 weeks before perfusion. This type of lesion disrupted innervation of the dorsal and anterior two-thirds of the bulb, judging from the disappearance of anti-olfactory marker protein staining in these areas.

\section{Biochemical characterization of the $R B-8$ antigen}

Protein amounts were determined by the bicinchoninic acid method (Pierce Chemical Co.) using BSA as the standard (Smith et al., 1985).

\section{Purification of RB-8 IgG}

RB- 8 ascites was pooled and the IgG was precipitated by addition of saturated ammonium sulfate to a final concentration of $47 \%(\mathrm{vol} / \mathrm{vol})$, followed by centrifugation for $1 \times 10^{4} \mathrm{~g}$-min. The pellet was resuspended in PBS and dialyzed extensively against PBS containing $0.02 \%$ sodium azide. RB-8 IgG was affinity-purified on a goat anti-mouse IgG column (Sigma). After adsorption to the column, antibody was eluted with 0.1 M glycine, pH 2.5, neutralized with $\mathrm{NaOH}$, and precipitated with $50 \%$ saturated ammonium sulfate. The pellet was resuspended to $1.6 \mathrm{mg} / \mathrm{ml}$ in PBS, cleared of particulate matter by centrifugation, and then dialyzed against PBS. Analysis of this preparation on 7-15\% discontinuous PAGESDS gels (see below) indicated that the vast proportion of the protein consisted of unmodified IgG heavy and light chains.

\section{Iodination of $R B-8 \operatorname{Ig} G$}

Purified RB-8 IgG was iodinated with carrier-free $\mathrm{Na}^{12 \zeta}$ I (New England Nuclear) using Iodogen- (Pierce Chemical Co.) coated vials according 
to the method of Salacinski et al. (1981). Unincorporated ${ }^{125}$ I was removed by passage over a Sephadex G-25 spin column (Tuszynski et al., 1980). In determining the specific activity of the labeled antibody, it was assumed that $88 \%$ of the ${ }^{125} \mathrm{I}$ IgG was recovered from the Sephadex G-25 column, since this degree of recovery was observed when already chromatographed ${ }^{125}$ I IgG was passed over this type of column.

\section{Preparation of membranes for binding assays}

Membranes were prepared from whole rat brain, including the olfactory bulb, the olfactory nerve and glomerular layers from the olfactory bulb (harvested by scraping the surface of frozen, intact bulbs), the remaining forebrain, and liver, spleen, muscle, thymus, and kidney. All steps were carried out on ice. The tissues were homogenized with a Potter-Elvehjem-type tissue grinder in 9 volumes of an ice-cold solution of 0.32 M sucrose in $5 \mathrm{~mm}$ sodium phosphate buffer, pH 7.2, $1 \mathrm{~mm}$ EDTA, pH 7.2 , and $1 \mathrm{~mm}$ phenylmethylsulfonyl fiuoride (PMSF). The pellet from a low-speed spin $\left(1.6 \times 10^{3} \mathrm{~g}\right.$-min) was rehomogenized in the buffered sucrose solution and recentrifuged, and the supernatants from these lowspeed spins were combined and centrifuged at $4 \times 10^{6} \mathrm{~g}$-min. The resulting pellet was resuspended in $0.01 \mathrm{M}$ Tris, $0.05 \mathrm{M} \mathrm{NaCl}, \mathbf{p H} 7.2$, and then subjected to a second, equivalent, high-speed spin, resuspended in Tris-saline at a final concentration of approximately $12 \mathrm{mg} / \mathrm{ml}$, and stored at $-70^{\circ} \mathrm{C}$.

\section{Direct RIA}

The binding of labeled RB-8 to membranes was assessed by a direct RIA, which utilized ${ }^{125}$ I-RB-8. Assay points were determined in triplicate. The ${ }^{125} \mathrm{I}-\mathrm{RB}-8$ was mixed with unlabeled RB-8 IgG at a ratio of $1: 3$ in order to decrease the specific activity. Binding was determined by incubating $100 \mu \mathrm{g}$ of membrane protein in a final volume of $200 \mu \mathrm{l}$ PBS-1\% BSA, with varying concentrations either of labeled antibody alone or with the addition of excess unlabeled IgG. The membranes were sedimented by centrifugation in an Eppendorf-type microcentrifuge (Beckman) for $10 \mathrm{~min}$, washed once in PBS-1\% BSA, pelleted again and counted. No additional binding sites were detected in the supernatants from this type of assay by higher speed centrifugation at $4 \times$ $10^{6} \mathrm{~g}$-min on a Beckman Airfuge.

\section{$S D S-P A G E$}

Samples of membranes from the whole brain, the olfactory and glomerular layers of the olfactory bulb, or the remaining forebrain were solubilized in SDS sample buffer containing $4 \mathrm{~mm}$ dithiothreitol, and run on discontinuous $7-12 \%$ polyacrylamide gradient gels following the method of Laemmli (1970).

\section{Electrophoretic transfers and immunoblots}

After separation by SDS-PAGE, proteins were electrophoretically transferred to nitrocellulose paper (Schleicher and Schuell; $0.45 \mu \mathrm{m}$ pore size) at $120 \mathrm{~V}$ for 4-5 hr, using a Bio-Rad Trans Blot cell according to the method of Towbin et al. (1979). After transfer, the paper was rinsed in PBS for $45 \mathrm{~min}$, and nonspecific immunoreactivity to proteins on the strips was blocked by incubation for $45 \mathrm{~min}$ in PBS with $20 \%$ normal horse serum. The nitrocellulose strips were incubated in the primary antibody for $1 \mathrm{hr}$. Pure IgG from RB- 8 ascites was used at a final concentration of $4 \mu \mathrm{g} / \mathrm{ml}$. Nonspecific binding was assessed by incubation in an equal concentration of IgG in normal mouse serum or of a nonreactive mouse monoclonal $\mathrm{IgG}_{1}$, designated IIDP-1 (the gift of Dr. J. Davie), which had been affinity-purified to homogeneity. The strips were subsequently washed in 3 changes of PBS with 5\% normal horse serum for a total of $30 \mathrm{~min}$, incubated for $30 \mathrm{~min}$ in biotinylated horse anti-mouse IgG at 1:200 in PBS-20\% normal horse serum, washed again, and incubated for $30 \mathrm{~min}$ with a complex of avidin and biotinylated HRP, as recommended by the manufacturer. The complex was visualized by reaction with $0.2 \%$ diaminobenzidine and $0.01 \%$ hydrogen peroxide in $0.1 \mathrm{~m}$ sodium citrate buffer, $\mathrm{pH} 5.1$, for $20-45 \mathrm{sec}$, rinsed in water, and allowed to air-dry.

\section{Results}

\section{Distribution of the $R B-8$ antigen \\ Olfactory epithelium}

In cryostat sections of the olfactory epithelium, staining with the monoclonal antibody RB-8 permits the division of the epithelium and the primary olfactory projection into 2 parts: 1 zone of the epithelium and its axons, which stain well with the monoclonal antibody, designated RB-8-positive; and 1 zone which stains poorly, if at all, with the antibody, designated RB8-negative. The RB-8-positive area includes the ventral and lateral epithelium, where there is intense staining of the fascicles of the olfactory nerve that project from this part of the epithelium, as well as some weaker staining of the epithelium itself (Fig. 1, $A, E$ ). In the epithelium, staining is concentrated in the middle third, where the olfactory neuron somata reside, and close examination of the original sections indicates that some slender apical processes, which resemble the dendrites of these sensory neurons, are stained. The olfactory nerve fascicles (also known as the fila olfactoria) that are densely stained with RB-8 are first apparent as thin bundles just deep to the basal lamina, which then coalesce with the large, densely stained bundles situated deep in the lamina propria. These larger fascicles also contain axons in passage from other, more rostral, parts of the epithelium.

In contrast, the $\mathrm{RB}-8$-negative area includes the dorsal recess and the tips of the dorsal turbinals (Fig. 1, $A, B$ ). The epithelial lining and the fine superficial axon fascicles that conduct fibers from this area are not stained. The larger fiber bundles deep to these RB-8-negative areas are stained, but to a much lighter degree than the ventrolateral fascicles. More anteriorly in the epithelium, where there should be less of a contribution of axons from RB-8-positive epithelium to these large bundles, their staining drops to near-background. When antibodies to olfactory marker protein and vimentin are used as general immunohistochemical markers for olfactory sensory neurons and axons, there is no differential staining of the RB-8-positive and negative areas of the epithelium or olfactory nerve. In counterstained sections, no histological differences are evident between these 2 areas of the epithelium. As the larger bundles of sensory cell axons coalesce near the olfactory bulb, fibers from the 2 areas tend to remain segregated from each other.

The boundary between the RB-8-positive and negative zones is relatively sharp (Fig. $1 D$ ), and can be identified by the transition in the staining of the olfactory epithelium and of the superficial bundles of olfactory axons situated just deep to the basal lamina. The degree of staining of the larger fascicles undergoes a more gradual change in this boundary region; the fascicles deep to the boundary region also contain fibers from more ventral and lateral parts of the epithelial sheet, on their way to the olfactory bulb.

The extent of the RB-8-positive and RB-8-negative areas was assessed in series of sections through the olfactory epithelium from 6 rats. The staining pattern and the boundaries on the turbinals and septum between RB-8-positive and negative areas were consistent and reproducible from animal to animal. Careful examination of these sections indicated that the unstained parts of the epithelium formed a single, contiguous zone. Caudal to the level illustrated in Figure 1, the unstained epithelium that lines the tips of the turbinals fuses with the epithelium that lines the dorsal recess. Rostral to the level shown in Figure 1, the unstained epithelium extends along the dorsal recess and along the tip of the most dorsal endoturbinal to the anterior boundary of the olfactory epithelium. These relationships are shown on the flattened map of the olfactory epithelium (Fig. 2). To summarize, the RB-8-negative zone occupies the posterior and dorsal parts of the epithelial sheet, constituting about one-third of the sheet; it is flanked by RB-8-positive zones, which constitute the remaining two-thirds.

\section{Olfactory bulb}

The distinction between RB-8-positive and negative zones of the olfactory epithelium is maintained in the projection of the epithelium onto the olfactory bulb. The olfactory nerve layer of the ventral and lateral part of the bulb is more densely stained with RB-8 than is the dorsal and medial part (Fig. $3 A$ ). Likewise, 

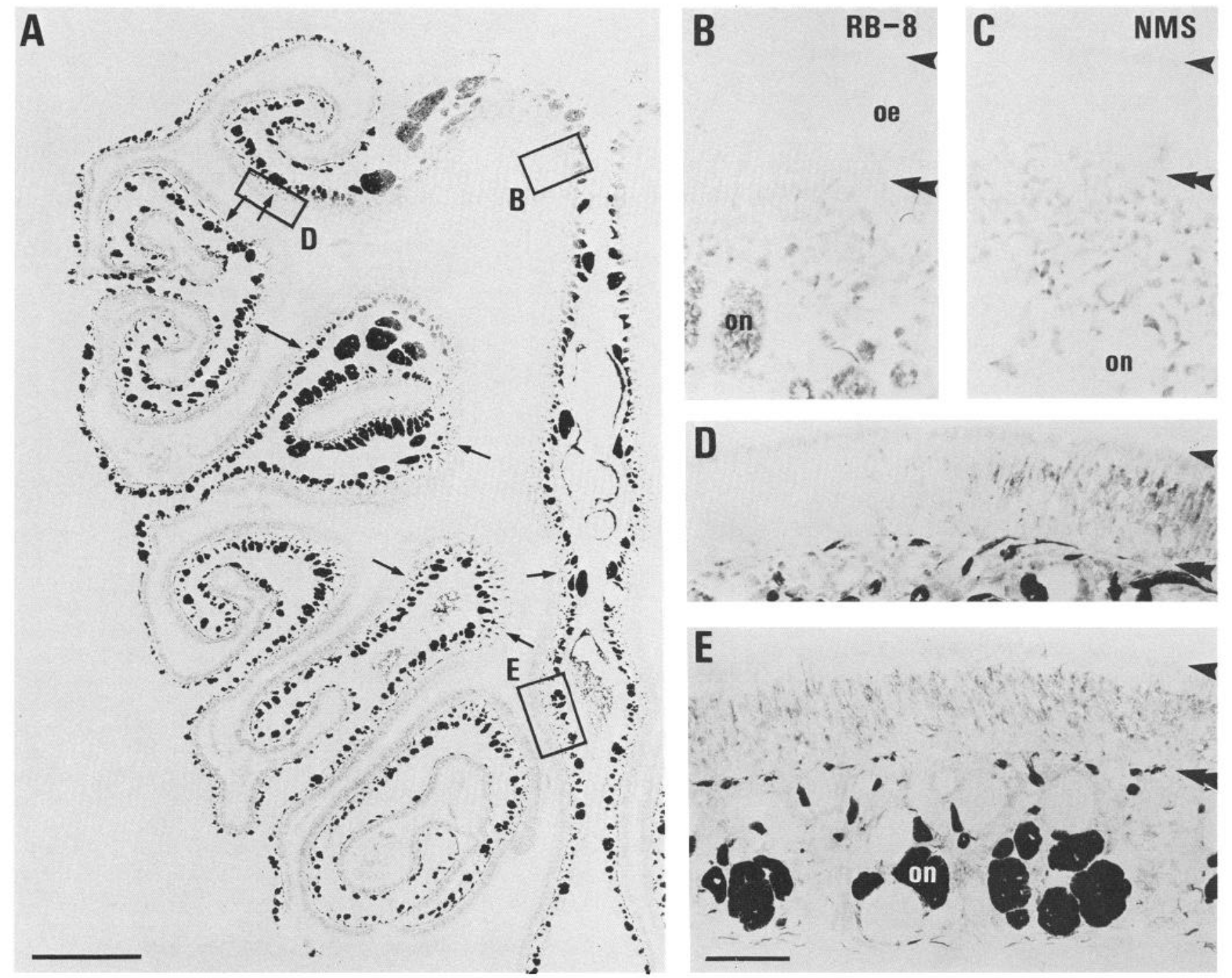

Figure 1. A, Coronal section of a decalcified rat muzzle stained with RB-8. In this photomicrograph, dorsal is up, and the nasal septum is at the right. The boxes indicate fields shown at higher power on the right. For purposes of comparison with Figure 2, the boundaries between RB-8positive (ventral and lateral) and RB-8-negative (dorsal recess and tips of the dorsal turbinates) parts of the epithelium are indicated by arrows in this low-power photomicrograph. B, RB-8-negative area, from the dorsal recess. The epithelium (oe) is unstained, and there is no staining of the fine olfactory nerve fascicles just below the basal lamina (double arrowhead). There is weak staining of the larger olfactory nerve fascicles (on) deeper in the lamina propria, compared to the normal mouse serum control $(C)$. Note that the staining is far weaker than in $E$. The single arrowhead indicates the apical surface of the epithelium. $C$, Area similar to $B$ from an adjacent section stained with normal mouse serum (NMS). $D$, RB-8 boundary zone, from the dorsal ectoturbinate. There is a relatively sharp transition in the staining of the epithelium. There is also a shift in the staining of the fascicles of the olfactory nerve just deep to the basal lamina, which is displaced medially (left in this photomicrograph) from the epithelial boundary by the course of these axons toward the olfactory bulb. $E$, RB-8-positive area, from the ventral septum. There is staining of the olfactory epithelium and heavy staining of the fascicles of the olfactory nerve (on) just below the basal lamina and larger fiber bundles deep in the lamina propria. Scale bars, $600 \mu \mathrm{m}$ in $A$ and $60 \mu \mathrm{m}$ for $B-E$.

in the glomerular layer, there is dense staining of the neuropil in glomeruli of the ventrolateral bulb (Fig. $3 D$ ), while the glomeruli of the dorsomedial bulb are unstained, relative to the normal mouse serum stained control, or are only very weakly stained (Fig. $3 B$ ).

In the positive glomeruli, RB-8 staining is superimposable on anti-olfactory marker protein labeling of the olfactory axons and their terminals, as is shown in double-labeled, frozen, semithin sections of the ventral olfactory bulb (Fig. 4). Since olfactory marker protein is limited to olfactory axons, its colocalization with RB- 8 would suggest that the RB-8 antigen is also found on these axons. Both antibodies leave unstained those parts of the glomerular neuropil that are apparently occupied by the other elements of the glomerulus. There is additional, indirect evidence that RB-8 stains olfactory axons and their terminals. Extracranial lesions of the olfactory nerve cause the staining with RB-8 to disappear from the olfactory glomeruli (Fig. 5). The degeneration of the olfactory axons and terminals within the glomeruli was verified by incubating an adjacent section with anti-olfactory marker protein, which left the denervated glomeruli unstained.

The glomeruli in normal animals left unstained by RB- 8 are innervated by olfactory axons; the axonal projection from the olfactory epithelium to this dorsomedial part of the bulb can be demonstrated with anti-olfactory marker protein or antivimentin staining of adjacent sections.

The distribution of glomerular staining with RB-8 is summarized on a flattened map of the glomerular layer of the bulb 


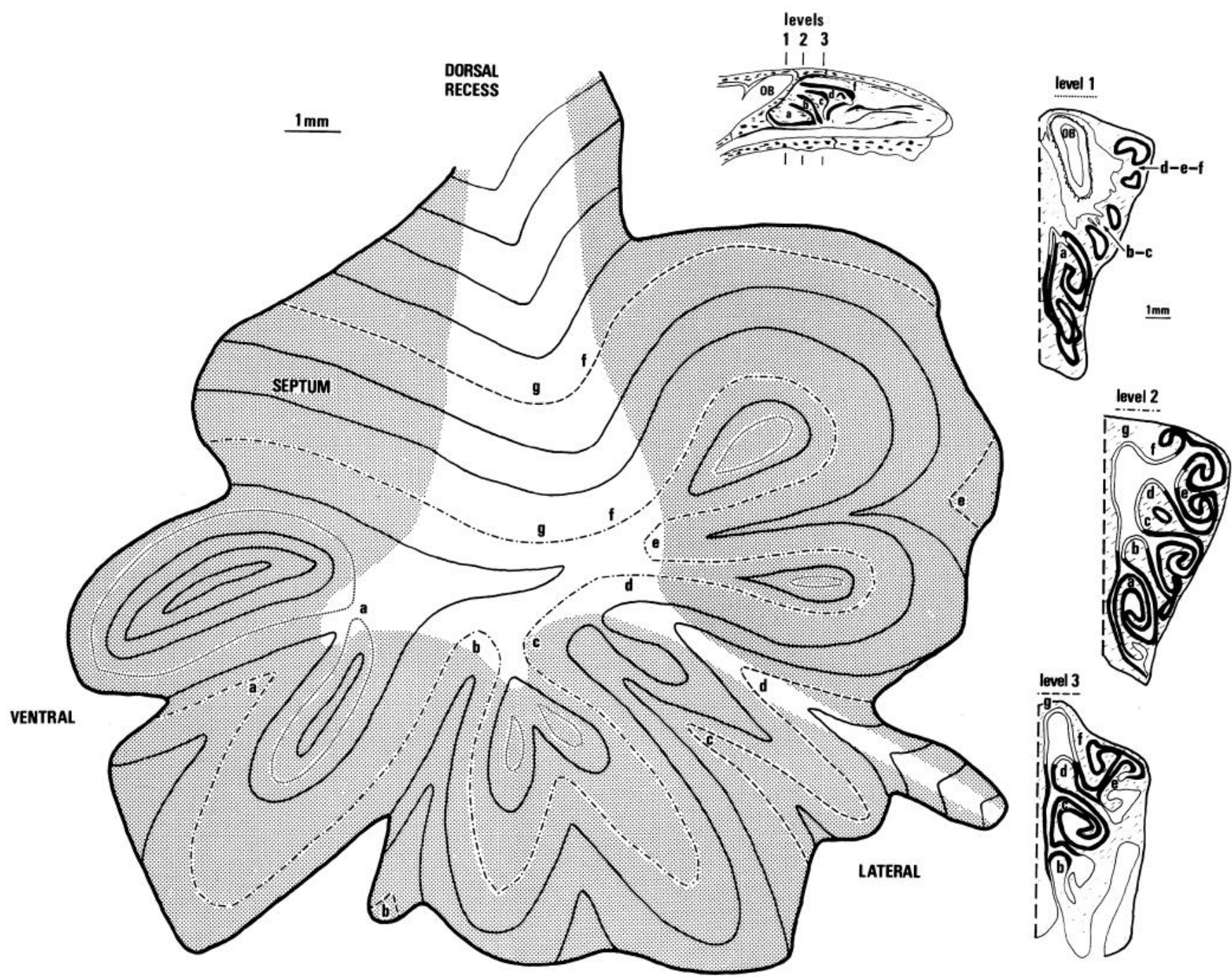

Figure 2. Two-dimensional reconstruction of RB-8 staining of the olfactory epithelium from a series of coronal sections. The details of construction are described in Materials and Methods. In the flattened reconstruction, shading indicates the RB-8-positive part of the epithelial sheet, while the unshaded area represents the RB-8-negative part. Each contour line maps the olfactory epithelium of a single coronal section. To illustrate the construction of the map, 3 representative sections (levels 1-3) are indicated on the map by different types of dashed lines. The corresponding coronal sections and the type of dashed line used to represent them are shown on the right. The anterior-posterior positions of levels 1-3 are indicated on the sagittal view of the rat nasal cavity at the top. In the coronal sections, the olfactory epithelium is represented by parallel lines; RB-8-positive areas of the epithelium are indicated by shading, and RB-8-negative areas are left blank. The letters on the map indicate the position of the various turbinates and correspond to the letters that designate the turbinates in the coronal sections and the sagittal view of the nasal cavity.

(Fig. 6). This type of map illustrates well the clustering of the unstained and lightly stained glomeruli in the dorsomedial part of the bulb, which is flanked by the densely stained glomeruli of the ventrolateral bulb. It should be noted that the segregation of the RB-8-negative and positive glomeruli in the bulb is not complete, in contrast to the relatively sharp boundary between the RB-8-negative and positive zones in the epithelium. For example, in the dorsomedial bulb, there are rare, densely stained glomeruli scattered among the unstained or lightly stained glomeruli, as shown on the map of the bulb in this region (Fig. 6). Likewise, on the medial and lateral sides of the bulb, unstained glomeruli are intermingled with heavily labeled glomeruli, and other glomeruli show an intermediate intensity of staining with RB-8 (Figs. $3 C$ and 6). Finally, in the transitional areas of the bulb, there are rare, nonuniformly stained glomeruli in which the projection to one part of the glomerulus is labeled, while the other part is not (Fig. 3C).

In the accessory olfactory system, the fascicles of the accessory olfactory nerve are uniformly and densely labeled with RB-8. Likewise, the glomeruli in the accessory olfactory bulb are densely and homogeneously stained.

\section{Distribution of the $R B-8$ antigen}

Two technical questions regarding the distribution of the RB- 8 antigen must be discussed. The first is whether the differential staining pattern indicates the differential distribution of an antigen, or whether the differences in staining might be due to some other cause. The second is whether the antigen is present on olfactory axons or associated supporting cells.

With regard to the first question: The division of the epithelium and the primary olfactory projection into RB-8-positive and RB-8-negative portions is likely to represent a real, biochemical difference between these parts of the epithelium and their axonal projection rather than an artifact of our methodology, since the staining pattern was consistently observed in each of the young rats we examined and was independent of 

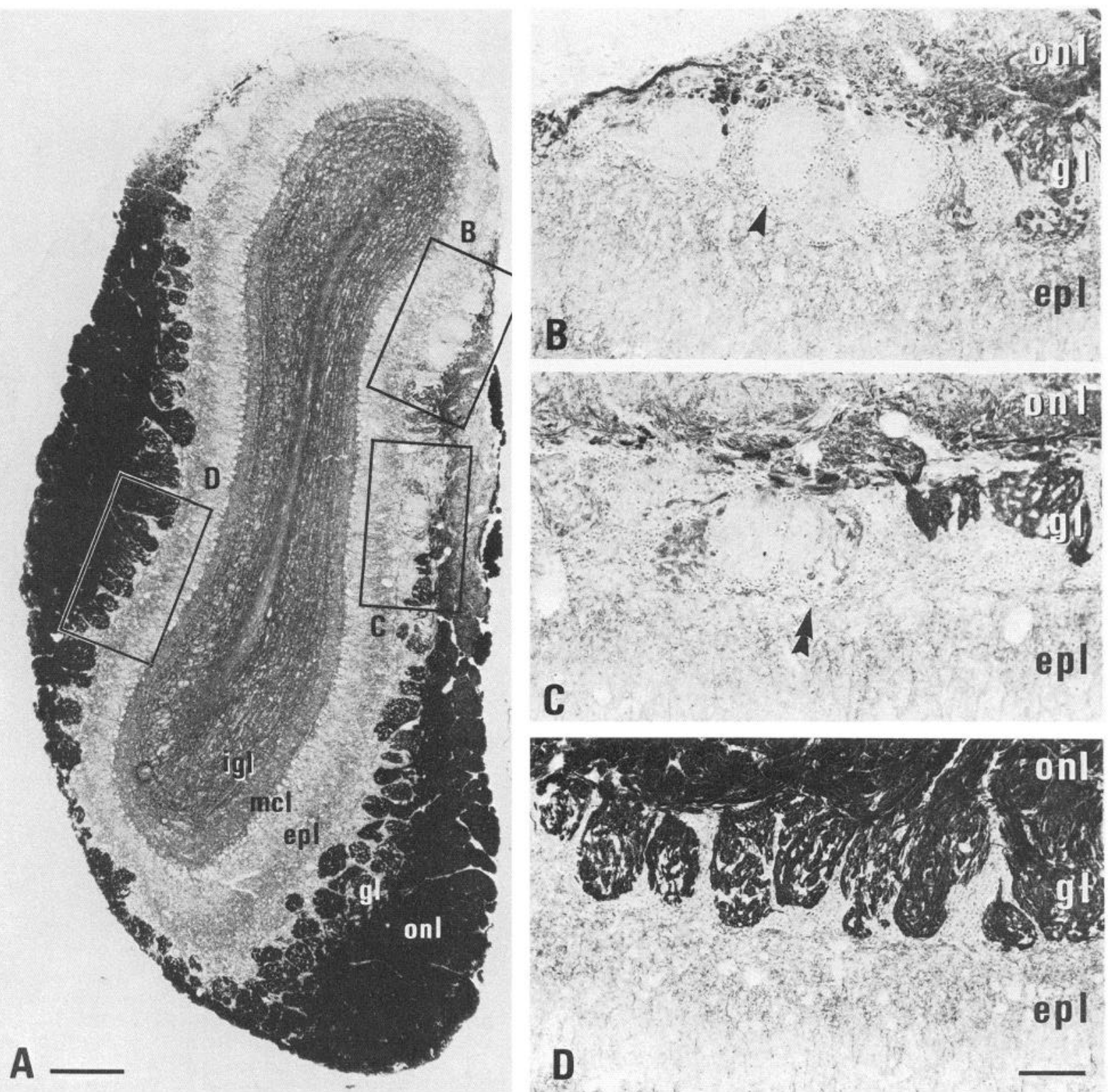

Figure 3. A, Coronal section of the olfactory bulb stained with RB-8. Boxes indicate the fields, which are shown at higher power to the right. Note that the olfactory nerve layer $(\mathrm{onl})$ and glomerular layer $(\mathrm{gl})$ in the ventral and lateral portion of the bulb are heavily stained, while the olfactory nerve layer in the dorsomedial olfactory bulb is more lightly stained. $B$. In the dorsomedial olfactory bulb, the glomeruli are either unstained or only lightly stained. The arrowhead indicates an unstained glomerulus. $C$, In the medial olfactory bulb, some glomeruli are unstained, while others show a moderate degree of labeling. The glomerulus indicated by the double arrowhead is unstained in its dorsal half (left), while moderately stained in its ventral half. $D$, In the lateral olfactory bulb, there is dense staining of the glomerular neuropil. External plexiform layer, epl; mitral cell layer, $m c l$; internal granular layer, $i g l$. Scale bars, $300 \mu \mathrm{m}$ in $A$ and $75 \mu \mathrm{m}$ for $B-D$.

the type of tissue fixation. Moreover, the lack of staining of the RB-8-negative part of the epithelium and olfactory projection is unlikely to reflect disease or damage of this area in some other, unspecified way, since there was no histological evidence of disease-indeed, no histological difference whatsoever. Neither was there a difference in the expression of 2 general olfactory markers, vimentin (Schwob et al., 1986) and olfactory marker protein (Hartman and Margolis, 1975; Margolis, 1972; Monti Graziadei et al., 1977), between the RB-8-negative and positive parts of the epithelium. The differential RB-8 staining pattern in the olfactory epithelium and the primary olfactory projection is established at an early stage of embryonic development (data not shown) and, hence, even less likely to result from some type of epithelial dysfunction that differentially affects RB-8-negative or RB-8-positive zones.

The weight of several lines of evidence suggests that the RB-8 antigen is indeed found in olfactory sensory neurons. First, the olfactory sensory neurons that are the cells of origin of this projection appear to be labeled, since the epithelial staining in the RB-8-positive areas, although weaker than the staining of the fascicles of the olfactory nerve, is limited to the middle layer of the epithelium, where the neuronal somata are located. In addition, stained apical dendritic processes can occasionally be seen. Second, glomerular labeling with RB-8 colocalizes with the distribution of olfactory marker protein, which, in the glomeruli, is found exclusively in olfactory axons and terminals 

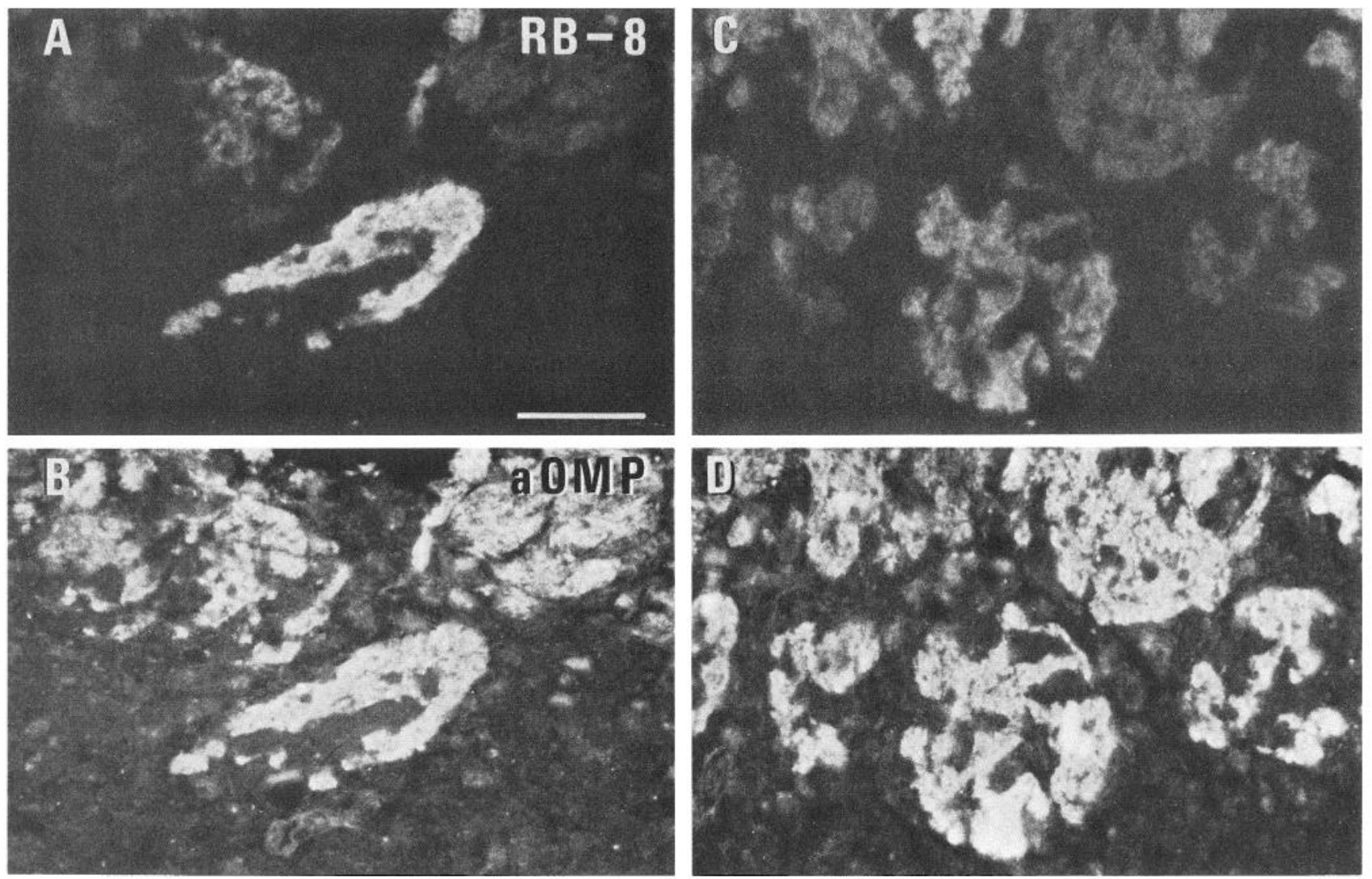

Figure 4. Colocalization of RB-8 and anti-olfactory marker protein staining in a double-labeled semithin frozen section. For each of 2 separate fields $(A, B$ and $C, D)$, the staining of glomeruli with RB-8 $(A, C)$ and antiserum to olfactory marker protein $(a O M P)(B, D)$ are shown. In each of the glomeruli, staining with the 2 antibodies is superimposable. Scale bar, $30 \mu \mathrm{m}$.

(Hartman and Margolis, 1975; Margolis, 1972; Monti Graziadei et al., 1977). The resolution of this double-labeling method is improved by using frozen semithin sections, and their use reduces the risk of identifying as identical those processes that are actually overlapping or closely associated. Third, the glomerular staining with RB-8 disappears as olfactory axons degenerate after lesions of the olfactory nerve. The alternative hypothesisthat the RB-8 antigen is localized to a closely associated supporting cell, rather than to the olfactory axons themselvescannot be fully excluded without direct electron-microscopic identification of the labeled processes. However, it is a less satisfactory explanation for these results and fits less well with published electron-microscopic observations. For example, the olfactory axons are arranged in bundles which are ensheathed by supporting cell processes as a whole rather than as individual axons (De Lorenzo, 1957; Gasser, 1956). Moreover, as these bundles enter the glomeruli, they lose their glial cell ensheathment (Andres, 1965; Berger, 1971; Doucette, 1984; Pinching and Powell, 1971; Raisman, 1985), and, in the glomerulus, the astrocytic processes are more closely associated with dendrites than with the olfactory axons (Pinching and Powell, 1971). In addition, glial cell hypertrophy, rather than degeneration, is the morphological response associated with olfactory nerve lesion (Berger, 1971; Doucette et al., 1983; Graziadei and Monti Graziadei, 1980; Pinching and Powell, 1972). These results are also the most consistent with our hypothesis that the RB- 8 antigen is found on the olfactory axons in the RB-8-positive part of the olfactory projection.

\section{Survey of CNS, PNS, and peripheral organs}

Elsewhere in the CNS, staining with RB-8 is widespread, but it is neither homogeneous nor universal. The RB-8 antigen is ev- ident in many areas of the neuropil. For example, the neuropil of the internal granular layer of the olfactory bulb is moderately stained with RB-8 (Fig. $3 A$ ). Most areas of the CNS show staining of about the same intensity as this layer of the bulb. However, there are several structures where the neuropil is unstained, such as CA 1 of the dorsal hippocampus (CA 1 of the ventral hippocampus does stain). Conversely, there are zones where the reaction product is strikingly more intense, such as layer IV of the medial entorhinal area. Neuronal and glial cell bodies (such as the mitral cells) do not appear to stain (Fig. 3A). Myelinated fiber tracts stain (but more weakly than the neuropil) in what appears to be an axon-like pattern, based on comparisons with the distribution of neurofilament proteins and Thy- 1 .

In the PNS, autonomic ganglia, including the superior cervical and superior mesenteric ganglia, and autonomic nerves are densely stained. At the light-microscopic level, it is difficult to resolve whether this staining is of axons or of closely associated Schwann cells. There is little or no staining of spinal nerves. Other peripheral organs, including liver, spleen, kidney, thymus, skeletal muscle, lung, and small intestine, have been surveyed. While the autonomic innervation to these organs is stained with RB-8, no parenchymal staining has been seen.

\section{Biochemical characterization of the $R B-8$ antigen}

\section{Characterization by $R I A$}

The antigen recognized by RB-8 was partially characterized by use of a direct RIA. The source of the antigen was whole brain, rather than the olfactory nerve, because of abundance and ease of preparation. Evidence that the antigen in whole brain is similar or identical to the antigen in the olfactory nerve will be presented. 

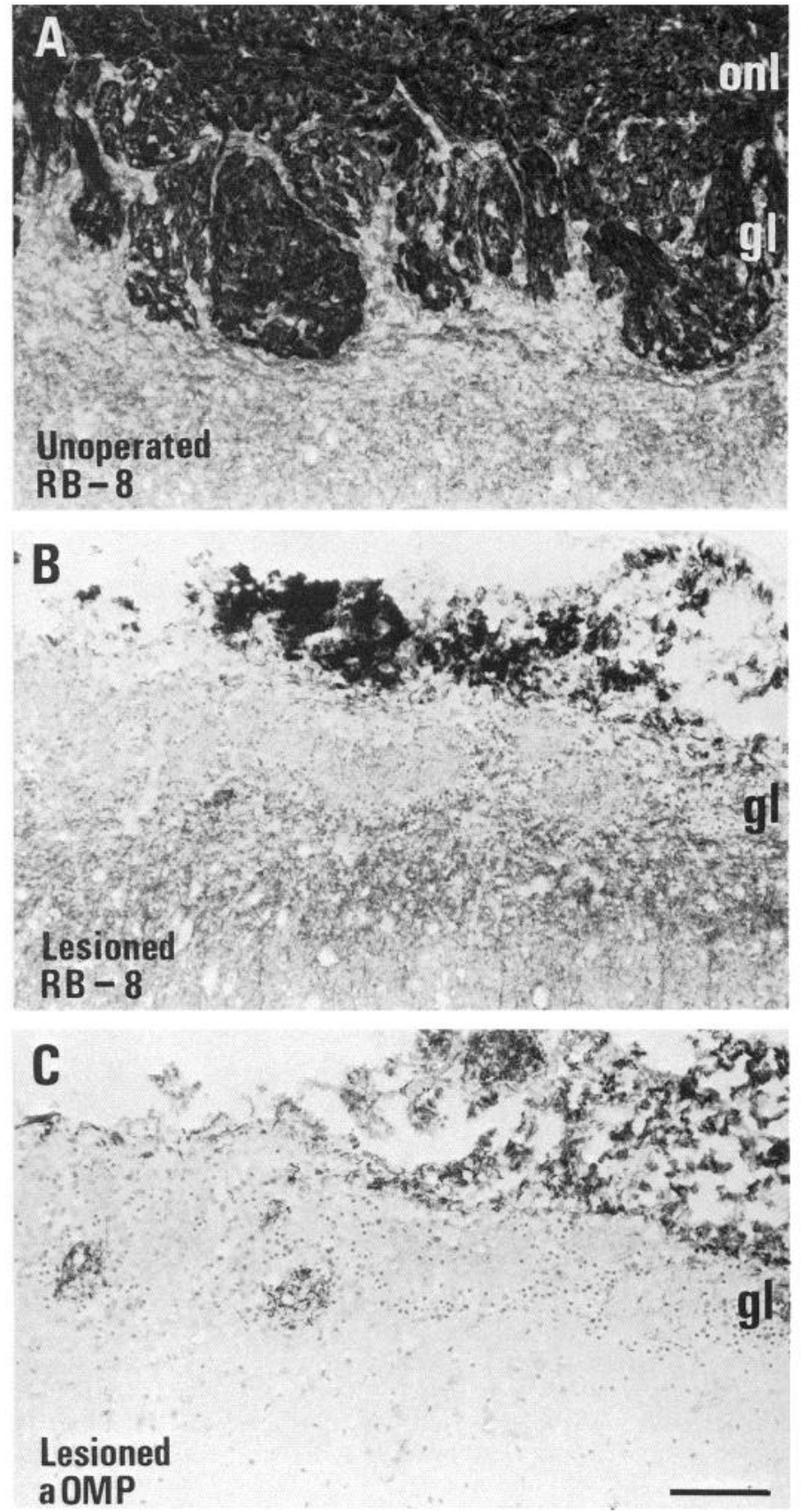

Figure 5. Disappearance of RB-8 and anti-olfactory marker protein staining after unilateral olfactory nerve lesion in an adult rat. $A$, Glomerular layer of the unoperated side, section stained with RB-8. B, Lesioned side, area of the olfactory bulb equivalent to that illustrated in $A$, stained with RB-8. Note lack of staining in glomeruli. $C$, Lesioned side, same area as in $B$ in an adjacent section stained with antiserum to olfactory marker protein $(a O M P)$. Note lack of staining. Scale bar, $75 \mu \mathrm{m}$.

The RB-8 antigen is membrane-associated. After washing membranes up to 6 times with hypotonic Tris-saline buffer, binding sites for radiolabeled RB-8 antibody remain pelletable by centrifugation (data not shown). The RB- 8 binding sites are solubilized from brain membranes by incubation in Tris-saline buffer containing $35 \mathrm{~mm}$ octylglucoside detergent, and can be recovered from the detergent supernatant by immobilization on nitrocellulose membranes (data not shown).

The binding of the radiolabeled antibody to membranes is
Table 1. Immunological specificity of RB-8 binding

\begin{tabular}{lcc} 
Antibody mixture & $\begin{array}{l}\text { Binding to whole- } \\
\text { brain membranes } \\
\text { (mean cpm } \pm \text { SD) }\end{array}$ & $\begin{array}{l}\text { Percent } \\
\text { of control }\end{array}$ \\
\hline${ }^{125}$ I-RB-8 alone & $15,986 \pm 828$ & 100 \\
+ RB-8 & $581 \pm 69$ & 4 \\
+ RB-5 & $16,233 \pm 957$ & 101 \\
+ RB-6 & $15,722 \pm 307$ & 98 \\
+ RB-7 & $16,072 \pm 105$ & 101 \\
+ RB-9 & $16,510 \pm 560$ & 103 \\
+ RB-10 & $16,311 \pm 298$ & 102 \\
+ RB-11 & $16,445 \pm 147$ & 103 \\
+ NMS & $16,456 \pm 226$ & 103
\end{tabular}

The effect of excess unlabeled homologous or heterologous IgG on binding of labeled RB-8 to whole-brain membranes was assayed as described in Materials and Methods. Labeled RB-8 was present at a saturating concentration of $1.3 \times$ $10^{-9}$. The effect of homologous antibody was assessed by adding excess unlabeled RB-8 IgG at $2.7 \times 10^{-7} \mathrm{M}$ per tube. The heterologous mouse monoclonal IgGs (RB-5-RB-11) and normal mouse serum (NMS) were used as dilutions of ascites or serum to give approximately $2.7 \times 10^{-7} \mathrm{M}$ of unlabeled mouse IgG per tube.

saturable and has a high affinity, as was shown when membranes were incubated with increasing concentrations of antibody (Fig. 7). Most of the bound counts can be displaced by excess unlabeled RB-8, which was added at a concentration of 200 times saturation. The residual counts represent nonspecifically bound antibody and can be subtracted from the total binding to give the specifically bound counts. The concentration of $\mathrm{IgG}$ at halfsaturation is $5.3 \times 10^{-10} \mathrm{M}$. While it is inappropriate to calculate an exact affinity constant from this figure, the value is consistent with a high-affinity binding process. At saturation, immunoglobulin binds at about $0.8 \mathrm{pmol} / \mathrm{mg}$ of membrane protein. This cannot be considered an exact determination of the concentration of the RB- 8 antigen, since binding of the labeled antibody could be univalent, bivalent, or a mixture of both. This value does, however, serve as a rough estimate of the amount of antigen; it demonstrates that the antigen is relatively rare and is present within the range of concentration of many neurotransmitter receptors.

At saturating concentrations of RB-8, heterologous, unlabeled IgGs have no effect on the binding of labeled antibody to wholebrain membranes (Table 1). The heterologous immunoglobulins were derived from a panel of monoclonal antibodies and from normal mouse serum; they were used at approximately 200 times the saturating concentration of RB-8. Since labeled RB-8 can be displaced only by cold RB- 8 , the most likely interpretation of these binding studies is that RB- 8 binds to a specific antigen in the brain membranes, rather than to a general immunoglobulin binding site.

RB-8 binding sites are sensitive to trypsin digestion (Table 2). Incubation of membranes at $37^{\circ} \mathrm{C}$ with active trypsin (which is subsequently inactivated with soybean trypsin inhibitor) diminishes specific binding to $7 \%$ of the value for untreated, incubated membranes. Treatment of membranes with trypsin that has been previously inactivated with inhibitor, or treatment with inhibitor alone, has essentially no effect on binding. This implies that the RB-8 antigen is a protein or a protein-linked moiety.

Other tissues were surveyed for the presence of the RB- 8 antigen (Table 3). Membranes from liver, muscle, spleen, kidney, and thymus have a specific binding of less than $1 \%$ of the antibody that is bound by equivalent amounts of brain membrane. This result is consistent with the failure of RB-8 to label the parenchyma of these organs in immunohistochemically stained sections. 


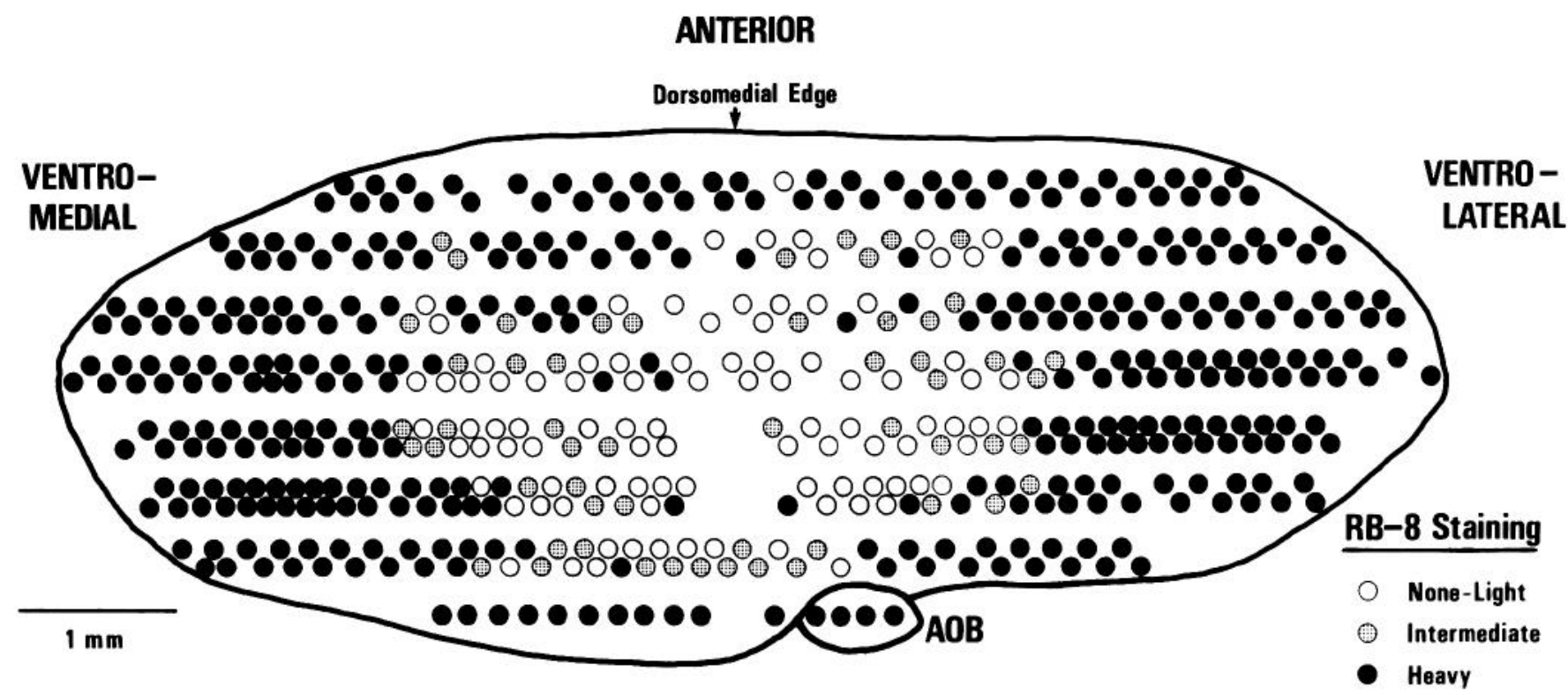

Figure 6. Glomerular labeling with RB-8, summarized on a 2-dimensional map of the glomerular layer of the olfactory bulb that was reconstructed from a series of coronal cryostat sections stained with the antibody. Each circle represents a glomerulus. The glomeruli from each single section are arranged in a double line for clarity of presentation. For each glomerulus, the intensity of staining with RB-8 is indicated by the key in the lower right. Glomeruli that are unstained, lightly stained, or moderately stained cluster in the dorsomedial part of the bulb and are flanked by the heavily stained glomeruli in the ventral and lateral parts of the bulb.

\section{Immunoblots}

Nitrocellulose blots of SDS-PAGE-separated membrane proteins from olfactory nerve and from forebrain minus the olfactory bulb were probed with purified $\mathrm{RB}-8 \mathrm{IgG}$, and gave an essentially identical pattern of immunoreactive proteins (Fig. 8). Immunoblots of both types of membranes show a prominent, specifically labeled band at $125 \mathrm{kDa} M_{\mathrm{r}}$. In mixtures of membranes from the forebrain and the olfactory nerve, there is only the single prominent immunoreactive band, which demonstrates the comigration in 1-dimensional SDS-PAGE gels of this protein from both types of membranes. It is unlikely that this band appears in the olfactory nerve membranes as a result of

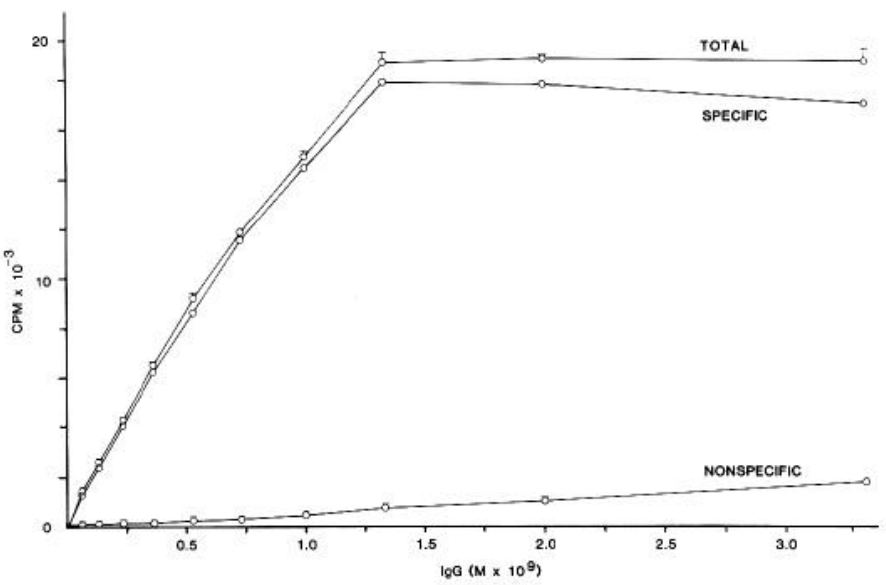

Figure 7. Binding of labeled RB- 8 to brain membranes. Varying concentrations of ${ }^{125} \mathrm{I}-\mathrm{RB}-8$ are added to brain membranes containing 100 $\mu \mathrm{g}$ of protein, either alone (Total) or in the presence of excess $(2.7 \times$ $10^{-7} \mathrm{~mm}$ ) unlabeled RB-8 IgG (Nonspecific). For these curves, each point is the mean and the bars show the standard deviation of 3 determinations. The nonspecific binding was subtracted from the total binding at each concentration of labeled IgG to give the amount of specifically bound IgG (Specific).

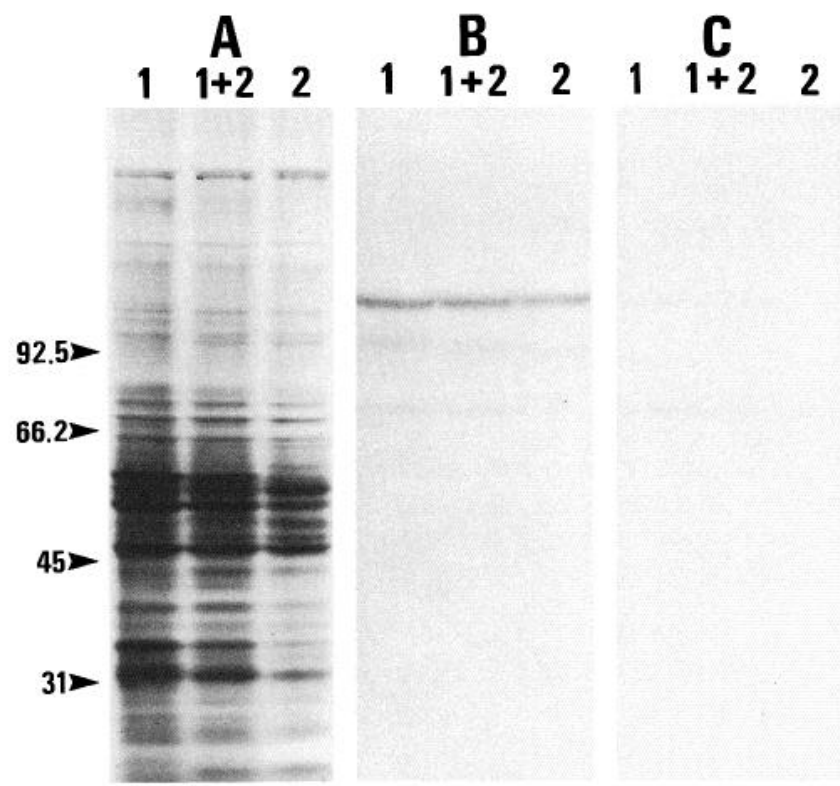

Figure 8. Immunoblot analysis of RB-8 antigen in olfactory nerve and brain membranes. SDS-PAGE 7-12\% gradient gel stained with Coomassie blue $(A)$ and posttransfer immunoblots stained with purified RB-8 $\mathrm{IgG}(B)$ or with an irrelevant $\mathrm{IgG}_{1}$ designated HDP-1 $(C)$. In $A-C$, lane 1 is $80 \mu \mathrm{g}$ of olfactory nerve membrane protein, lane 2 is $80 \mu \mathrm{g}$ of forebrain membrane protein, and lane $1+2$ is $40 \mu \mathrm{g}$ of olfactory nerve membrane protein and $40 \mu \mathrm{g}$ of forebrain membrane protein. The immunoreactive band at $125 \mathrm{kDa} M_{t}$ is present in membranes from both sources and comigrates in the mixing experiment. There are very much fainter bands at lower molecular weights, some of which are nonspecifically stained, compared with the original blots. The numbers along the left side designate the molecular weight standards (in $\mathrm{kDa}$ ) loaded in an adjacent lane; from top to bottom they are phosphorylase b, BSA, ovalbumin, and carbonic anhydrase. 
Table 2. Trypsin sensitivity of RB-8 binding site(s)

\begin{tabular}{lccrr}
\multicolumn{4}{c}{ Binding to whole-brain membranes } \\
\cline { 2 - 5 } Incubation conditions & $\begin{array}{l}\text { 125I-RB-8 alone } \\
\text { (mean cpm } \pm \text { SD) }\end{array}$ & + RB-8 & $\begin{array}{l}\text { Specific } \\
\text { binding }\end{array}$ & $\begin{array}{l}\text { Percent } \\
\text { of control }\end{array}$ \\
\hline Control & $15,091 \pm 531$ & $494 \pm 67$ & 14,597 & 100 \\
Trypsin followed by inhibitor & $1483 \pm 76$ & $403 \pm 45$ & 1080 & 7 \\
Trypsin + inhibitor & $14,622 \pm 74$ & $558 \pm 52$ & 14,054 & 92 \\
Inhibitor alone & $14,316 \pm 335$ & $541 \pm 34$ & 13,775 & 94
\end{tabular}

The direct RIA was modified to assess the trypsin sensitivity of RB-8 binding sites. Before addition of labeled RB-8, brain membranes containing $100 \mu \mathrm{g}$ of protein were incubated for $45 \mathrm{~min}$ at $37^{\circ} \mathrm{C}$ alone (Control), for $30 \mathrm{~min}$ with $0.17 \%$ trypsin, and then for $15 \mathrm{~min}$ with $0.24 \%$ soybean trypsin inhibitor (Trypsin followed by inhibitor), for $45 \mathrm{~min}$ with trypsin that had already been inactivated by addition of soybean trypsin inhibitor (Trypsin + inhibitor), or for 45 min with soybean trypsin inhibitor alone (Inhibitor alone). Labeled RB-8 was added at $1.3 \times 10^{-9} \mathrm{M}$ alone to determine total binding (125I-RB-8 alone). Nonspecific binding was determined by incubation in the presence of excess unlabeled RB-8 at $2.7 \times 10^{-7} \mathrm{M}(+\mathrm{RB}-8)$. Nonspecific binding was subtracted from total binding to give the specifically bound counts.

contamination of the olfactory nerve membrane preparation with membranes from deeper layers of the bulb, since the dissection was confined to the olfactory nerve and glomerular layers, as verified by direct histological examination. In addition to the prominent band at $125 \mathrm{kDa} M_{\mathrm{r}}$, some fainter, specifically immunoreactive, bands at lower molecular weights are present in both forebrain and olfactory nerve membranes. The significance of these bands and their relation to the $125 \mathrm{kDa}$ protein remain uncertain at the present time. A more detailed molecular characterization of the RB-8 antigen has yet to be completed. We have preliminary evidence that the antigen can be partially purified from brain membranes by detergent solubilization and passage over an RB-8 immunoaffinity column (data not shown). Hence, further characterization of the antigen should be feasible.

\section{Discussion}

The major finding of this work is that the primary olfactory projection is divided into 2 biochemically distinct zones. The primary olfactory projection consists of the olfactory epithelium, containing the olfactory sensory neurons and their axons, which leave the epithelium and travel to the olfactory bulb, where they form synapses. The monoclonal antibody RB-8 stains a contiguous zone equal to approximately two-thirds of the area of the primary projection. The other third is either not stained or stained at a much lower level. The antigen recognized by RB- 8 is bound to membranes and degraded by trypsin. On SDSPAGE, it migrates as a $125 \mathrm{kDa} M_{\mathrm{r}}$ protein.

There is good agreement between the RB- 8 staining pattern in the olfactory bulb and what is known from neuroanatomical

\section{Table 3. Tissue distribution of RB-8 antigen}

\begin{tabular}{lccrc}
\multicolumn{5}{l}{ Binding to membranes } \\
\cline { 2 - 5 } Tissue & $\begin{array}{l}\text { 12sI-RB-8 alone } \\
\text { (mean cpm } \pm\end{array}$ & + RB-8 & $\begin{array}{l}\text { Specific } \\
\text { binding }\end{array}$ & $\begin{array}{l}\text { Per- } \\
\text { cent of } \\
\text { brain }\end{array}$ \\
source & SD) & $537 \pm 87$ & 10,541 & 100 \\
Brain & $11,078 \pm 80$ & $590 \pm 61$ & 42 & $<1$ \\
Liver & $632 \pm 82$ & 35 & $<1$ \\
Muscle & $446 \pm 63$ & $411 \pm 60$ & 50 & $<1$ \\
Spleen & $492 \pm 19$ & $442 \pm 22$ & -18 & 0 \\
Kidney & $591 \pm 125$ & $609 \pm 47$ & 36 & $<1$ \\
Thymus & $595 \pm 56$ & $559 \pm 82$ &
\end{tabular}

The binding of labeled RB- 8 to membranes harvested from a variety of tissues was assayed in the usual manner. For each tissue source, membranes containing $100 \mu \mathrm{g}$ of protein were used. Total (125I-RB-8 alone), nonspecific (+RB-8), and specific bindings were determined as for Table 2 . studies of the axonal projection of the epithelium onto the bulb (Clark, 1951, 1957; Costanzo and O'Connell, 1978, 1980; Land, 1973; Land and Shepherd, 1974; Land et al., 1970): The dorsal part of the epithelium, which is RB-8-negative, has been shown to project to the dorsal part of the bulb, where the glomeruli are unstained or only lightly stained with RB-8; conversely, the ventral part of the epithelium, which is RB-8-positive, has been shown to project onto the ventral part of the bulb, where the glomeruli are heavily stained with RB-8. The correlation of the axonal projection with immunohistochemical staining, therefore, suggests that the level of the RB-8 antigen is stable along the length of the olfactory axon, from the epithelium to the bulb, and does not switch from positive at the epithelium to negative in the bulb or vice versa. That rare RB-8-positive glomeruli are found in the RB-8-negative dorsal part of the bulb is not inconsistent with the regional character of the epithelial projection onto the bulb. In experiments that anterogradely label the axons from the dorsal part of the epithelium and their terminals in the bulb, there are rare, unlabeled glomeruli within the cluster of labeled glomeruli in the dorsal part of the bulb (Land, 1973; Land and Shcphcrd, 1974; Land ct al., 1970). Thesc unlabeled glomeruli apparently receive their innervation from some region of the epithelium other than the dorsal part, and may correspond to the dorsally situated RB-8-positive glomeruli described here. In a similar vein, we cannot exclude the formal possibility that some of the neurons or their axons in the RB-8-positive zone are unstained. Given the density of the staining, the number of these axons, if they exist, is likely to be small.

On the basis of electrophoretic mobility and immunohistochemical staining pattern, the RB- 8 antigen is clearly different from all but one of the antigens previously described in the olfactory system. In contrast to the RB- 8 antigen, a number of antigens are homogeneously distributed within the primary olfactory projection. These include the olfactory marker protein with $M_{\mathrm{r}}=18.5 \mathrm{kDa}$ (Hartman and Margolis, 1975; Margolis, 1972; Monti Graziadei et al., 1977), vimentin with $M_{\mathrm{r}}=55$ kDa (Schwob et al., 1986), the NILE protein with $M_{\mathrm{r}}=215$ $\mathrm{kDa}$ (Stallcup et al., 1985), as well as those antigens in the olfactory epithelium and fascicles of the olfactory nerve that are recognized by a panel of monoclonal antibodies raised against the epithelium but that have not been biochemically characterized (Hempstead and Morgan, 1985). Similarly, the protein recognized by RB-8 has a slightly slower electrophoretic mobility than the $120 \mathrm{kDa} M_{\mathrm{r}}$ form of N-CAM (Edelman et al., 1983), which we visualized in immunoblots by staining with anti-NCAM antiserum (the generous gift of Dr. J. Covault; Covault and Sanes, 1985); this antiserum also stains the olfactory projection in a homogeneous and uniform manner (data not shown). Hence, the RB-8 antigen is unlikely to be N-CAM unless it is 
a rare variant of $\mathrm{nCAM}$, which has a distinctive electrophoretic mobility and staining pattern.

Two monoclonal antibodies that stain components of the olfactory projection in a nonhomogeneous fashion have recently been described. The first of these, designated 2B8, was generated against membranes of PC12 cells (Allen and Akeson, 1985a, b); well-stained olfactory sensory neurons were found scattered throughout the epithelial sheet, while, in the olfactory bulb, the staining intensity varied among the glomeruli. The antibody recognized proteins of 215 and $142 \mathrm{kDa} M_{\mathrm{r}}$ in the olfactory epithelium and bulb. A detailed map of the staining pattern in the olfactory bulb was not published; however, these antigens can be distinguished from the RB- 8 antigen by their molecular weight and the intense quality of the epithelial staining. In the rabbit olfactory system, Mori and his colleagues (Fujita et al., 1985; Mori et al., 1985) also described a nonhomogeneously distributed antigen recognized by a monoclonal antibody, designated R4B12. The staining pattern in the fascicles of the olfactory nerve and in the glomeruli of the bulb shows striking similarities to that of $\mathrm{RB}-8$, which suggests that the 2 antigens are the same. However, monoclonal antibody R4B12 (which was generated by immunization with rabbit olfactory bulb) does not cross-react with rat tissues, and the R4B12 antigen has not been characterized biochemically, making direct comparison impossible. Whether the antigens are identical or not, it is noteworthy, and may be taken as an indication of its potential importance, that this division of the primary olfactory projection into ventrolateral and dorsomedial parts is preserved in separate taxonomic orders.

At least 3 possible roles for the RB-8 antigen can be postulated. The first is a direct involvement in the olfactory transduction process. This seems highly unlikely, since the antigen is not detectable on the olfactory cilia where odorant receptors are localized (Adamek et al., 1984; Ottoson, 1956), but is concentrated in axonal and synaptic regions far from the site of sensory transduction. Furthermore, the antigen is found in many regions of the nervous system besides the primary olfactory projection. An alternative possibility is that the RB-8 antigen is involved in some aspect of neurotransmitter function. The neurotransmitter(s) used at the olfactory sensory neuron-mitral cell synapse is, at present, unknown. Perhaps the olfactory epithelium is divided into zones that use distinct neurotransmitters and the RB-8 antigen plays some as-yet-unknown role in one of these. A third possibility relates to the development of the primary olfactory projection. Since the RB-8 antigen is restricted to two-thirds of the total projection, and since the projection has some topographic specificity along the dorsoventral axis of the bulb and epithelium, it is possible that the RB-8 antigen is involved in directing axons to their final destination in the bulb. Since the RB-8-positive zonc is so broad, the antigen could not ensure connectional specificity by itself. However, it might be involved in directing fibers to a broad zone in which other molecular species would take over the task of directing the axons to their precise final destination.

Whatever the role of the RB- 8 antigen, it is possible that the boundary between the RB-8-positive and RB-8-negative zones is functionally relevant for sensory coding by the olfactory epithelium and bulb. It should be noted that there is a spatial component to the receptivity and coding of olfactory stimuli (reviewed by Moulton, 1976). In the olfactory epithelium, odorant-specific regional differences have been demonstrated electrophysiologically in nonmammalian vertebrates (Mackay-Sim et al., 1982) and by the uptake of radiolabeled 2-deoxyglucose in mammals (Lancet et al., 1981); these differences imply a nonhomogeneous distribution of odorant-specific (or selective) sensory neurons. Given that the spatial relations between broad areas of the olfactory epithelium are maintained in the axonal projection onto the bulb, it is not surprising that odorants ac- tivate glomeruli in a punctate and regional manner when assessed electrophysiologically (Adrian, 1953, 1954) or by 2-deoxyglucose uptake (Benson et al., 1985; Jourdan et al., 1980; Lancet et al., 1982; Skeen, 1977; Stewart et al., 1979). The functional relevance of the border established by RB-8 is amenable to experimental analysis. For example, one may hypothesize that glomeruli that are maximally activated by a given odorant are wholly RB-8-negative (or vice versa), and it would be possible to directly test such a hypothesis by comparing, in the same animal, the pattern of metabolic activation in the glomerular layer of the bulb with the RB-8 staining pattern. Although all odorants need not respect this boundary, the finding that some odorants do respect the RB-8-defined border would further implicate this division of the primary olfactory projection as of fundamental importance in the coding of olfactory information.

\section{References}

Adamek, G. D., R. C. Gesteland, R. G. Mair, and B. Oakley (1984) Transduction physiology of olfactory receptor cilia. Brain Res. 310 . 87-97.

Adrian, E. D. (1953) Sensory messages and sensations: The response of the olfactory organs to different smells. Acta Physiol. Scand. 29: 5-14.

Adrian, E. D. (1954) The basis of sensations: Some recent studies of olfaction. Br. Med. J. 1: 287-290.

Allen, W. K., and R. Akeson (1985a) Identification of a cell surface glycoprotein family of olfactory receptor neurons with a monoclonal antibody. J. Neurosci. 5: 284-296.

Allen, W. K., and R. Akeson (1985b) Identification of an olfactory receptor neuron subclass: Cellular and molecular analysis during development. Dev. Biol. 109: 393-401.

Andres, K. H. (1965) Der Feinbau des Bulbus olfactorius der Ratte unteer besonderer Berucksichtigung der synaptischen Verbindungen. Z. Zellforsch. 65: 530-561.

Benson, T. E., G. D. Burd, C. A. Greer, D. M. D. Landis, and G. M. Shepherd (1985) High-resolution 2-deoxyglucose autoradiography in quick-frozen slabs of neonatal rat olfactory bulb. Brain Res. 339: 67-78.

Berger, B. (1971) Formes diverse de degenerescence des boutons synaptiques dans le glomerule olfactif de lapin apres lesion du nerf olfactif. Brain Res. 33: 218-222.

Clark, W. E. L. (1951) The projection of the olfactory epithelium on the olfactory bulb in the rabbit. J. Neurol. Neurosurg. Psychiatry 14 . $1-10$.

Clark, W. E. L. (1957) Inquiries into the anatomical basis of olfactory discrimination. Proc. R. Soc. Lond. [Biol.] 146: 299-319.

Costanzo, R. M., and R. J. O'Connell (1978) Spatially organized projections of hamster olfactory nerves. Brain Res. 139: 327-332.

Costanzo, R. M., and R. J. O'Connell (1980) Receptive fields of second-order neurons in the olfactory bulb of the hamster. J. Gen. Physiol. 76: 53-68.

Covault, J., and J. R. Sanes (1985) Neural cell adhesion molecule (NCAM) accumulates in denervated and paralyzed skeletal muscles. Proc. Natl. Acad. Sci. USA 82: 4544-4548.

De Lorenzo, A. J. (1957) Electron microscopic observations of the olfactory mucosa and olfactory nerve. J. Biophys. Biochem. Cytol. 3: $839-850$

Doucette, J. R. (1984) The glial cells in the nerve fiber layer of the rat olfactory bulb. Anat. Rec. 210: 385-391.

Doucette, J. R., J. A. Kiernan, and B. A. Flumerfelt (1983) The reinnervation of olfactory glomeruli following transection of primary olfactory axons in the central or peripheral nervous system. J. Anat. 137: 1-19.

Edelman, G. M., S. Hoffman, C.-M. Chuong, J.-P. Thiery, R. Brackenbury, W. J. Gallin, M. Grumet, M. E. Greenberg, J. J. Hemperly, C. Cohen, and B. A. Cunningham (1983) Structure and modulation of neural cell adhesion molecules in early and late embryogenesis. Cold Spring Harbor Symp. Quant. Biol. 48: 515-526.

Fujita, S. C., K. Mori, K. Imamura, and K. Obata (1985) Subclasses of olfactory receptor cells and their segregated central projections demonstrated by a monoclonal antibody. Brain Res. 326: 192-196.

Galfre, G., S. C. Howe, C. Milstein, G. W. Butcher, and J. C. Howard 
(1977) Antibodies to major histocompatibility antigens produced by hybrid cell lines. Nature 266: 550-552.

Gasser, H. S. (1956) Olfactory nerve fibers. J. Gen. Physiol. 39: 473498.

Graziadei, P. P. C., and G. A. Monti Graziadei (1980) Neurogenesis and neuron regeneration in the olfactory system of mammals. III. Deafferentation and reinnervation of the olfactory bulb following section of the fila olfactoria in rat. J. Neurocytol. 9: 145-162.

Hartman, B. K., and F. L. Margolis (1975) Immunofluorescence localization of the olfactory marker protein. Brain Res. 96: 176-180.

Hempstead, J. L., and J. I. Morgan (1985) A panel of monoclonal antibodies to the rat olfactory epithelium. J. Neurosci. 5: 438-449.

Johnson, G. D., and G. M. deC. Nogueria Araujo (1981) A simple method of reducing the fading of immunofluorescence during microscopy. J. Immunol. Methods 43: 349-350.

Jourdan, F., A. Duveau, I. Astic, and A. Holley (1980) Spatial distribution of [14C]2-deoxyglucose uptake in the olfactory bulbs of rats stimulated with two different odours. Brain Res. 188: 139-154.

Laemmli, U. K. (1970) Cleavage of structural proteins during the assembly of the head of bacteriophage T4. Nature 227:680-685.

Lancet, D. (1986) Vertebrate olfactory reception. Annu. Rev. Neurusci. 9: 329-355.

Lancet, D., J. S. Kauer, C. A. Greer, and G. M. Shepherd (1981) High resolution 2-deoxyglucose localization in olfactory epithelium. Chem. Senses 6: 343-349.

Lancet, D., C. A. Greer, J. S. Kauer, and G. M. Shepherd (1982) Mapping of odor-related neuronal activity in the olfactory bulb by high-resolution 2-deoxyglucose autoradiography. Proc. Natl. Acad. Sci. USA 79: 670-674.

Land, L. J. (1973) Localized projection of olfactory nerves to rabbit olfactory bulb. Brain Res. 63: 153-166.

Land, L. J., and G. M. Shepherd (1974) Autoradiographic analysis of olfactory receptor projections in the rabbit. Brain Res. 70: 506-510.

Land, L. J., R. P. Eagher, and G. M. Shepherd (1970) Olfactory nerve projections to the olfactory bulb in the rabbit: Demonstration by means of a simplified ammoniacal silver degeneration method. Brain Res. 23: 250-254.

Mackay-Sim, A., P. Shaman, and D. G. Moulton (1982) Topographic coding of olfactory quality: Odorant-specific patterns of epithelial responsivity in the salamander. J. Neurophysiol. 48: 584-596.

Margolis, F. L. (1972) A brain protein unique to the olfactory bulb. Proc. Natl. Acad. Sci. USA 69: 1221-1224.

McLean, I. W., and P. K. Nakane (1974) Periodate-lysine-paraformaldehyde fixative. A new fixative for immunoelectron microscopy. J. Histochem. Cytochem. 22: 1077-1083.

Monti Graziadei, G. A., F. L. Margolis, J. W. Harding, and P. P. C. Graziadei (1977) Immunocytochemistry of the olfactory marker protein. J. Histochem. Cytochem. 25: 1311-1316.

Mori, K., S. C. Fujita, K. Imamura, and K. Obata (1985) Immunohis- tochemical study of subclasses of olfactory nerve fibers and their projections to the olfactory bulb in the rabbit. J. Comp. Neurol. 242: 214-229.

Moulton, D. G. (1976) Spatial patterning of response to odors in the peripheral olfactory system. Physiol. Rev. 56: 578-593.

Mozell, M. M. (1964) Olfactory discrimination: Electrophysiological spatiotemporal basis. Science 143: 1336-1337.

Ottoson, D. (1956) Analysis of the electrical activity of the olfactory epithelium. Acta Physiol. Scand. [Suppl. 122] 35: 1-83.

Pinching, A. J., and T. P. S. Powell (1971) The neuropil of the glomeruli of the olfactory bulb. J. Cell Sci. 9: 347-377.

Pinching, A. J., and T. P. S. Powell (1972) A study of terminal degeneration in the olfactory bulb of the rat. J. Cell Sci. 10: 585-619.

Raisman, G. (1985) Specialized neuroglial arrangement may explain the capacity of vomeronasal axons to reinnervate central neurons. Neuroscience 14: 237-254.

Salacinski, P. R. P., C. McLean, J. E. C. Sykes, V. V. Clement-Jones, and P. J. Lowry (1981) Iodination of proteins, glycoproteins, and peptides using a solid-phase oxidizing agent, 1,3,4,6-tetrachloro-3a,6adiphenyl glycoluril (Iodogen). Anal. Biochem. 117: 136-146.

Schwob, J. E., and D. I. Gottlieb (1985) The primary olfactory projection has two chemically distinct zones. Soc. Neurosci. Abstr. 11: 970.

Schwob, J. E., N. B. Farber, and D. I. Gottlieb (1986) Neurons of the olfactory epithelium in adult rats contain vimentin. J. Neurosci. 6 : 208-217.

Skeen, L. C. (1977) Odor-induced patterns of deoxyglucose consumption in the olfactory bulb of the treeshrew, Tupaia glis. Brain Res. 124: 147-153.

Smith, P. K., R. I. Krohn, G. T. Hermanson, A. K. Mallia, F. H. Gartner, M. D. Provenzano, E. K. Fujimoto, N. M. Goeke, B. J. Olson, and D. C. Klenk (1985) Measurement of protein using bicinchoninic acid. Anal. Biochem. 150: 73-85.

Stallcup, W. B., L. L. Beasley, and J. M. Levine (1985) Antibody against nerve growth factor-inducible large external (NILE) glycoprotein labels nerve fiber tracts in the developing nervous system. $J$. Neurosci. 5: 1090-1101.

Stewart, W. B., J. S. Kauer, and G. M. Shepherd (1979) Functional organization of rat olfactory bulb analysed by the 2-deoxyglucose method. J. Comp. Neurol. 185: 715-734.

Towbin, H., T. Staehlin, and J. Gordon (1979) Electrophoretic transfer of proteins from polyacrylamide gels to nitrocellulose sheets: Procedure and some applications. Proc. Natl. Acad. Sci. USA 76: 43504354.

Tuszynski, G. P., L. Knight, J. R. Piperno, and P. N. Walsh (1980) A rapid method for removal of [ $\left.{ }^{125} I\right]$ iodide following iodination of protein solutions. Anal. Biochem. 106: 118-122.

Van Essen, D. C., and J. H. R. Maunsell (1980) Two-dimensional maps of the cerebral cortex. J. Comp. Neurol. 191: 255-281. 